\title{
Treatment and Pregnancy Outcomes of Pregnant Women Exposed to Second-line Anti-tuberculosis Drugs in South Africa
}

Idah Mokhele ( $\boldsymbol{\sigma}$ imokhele@heroza.org)

Health Economics and Epidemiology Research Office https://orcid.org/0000-0002-8054-0116

Nelly Jinga

Health Economics and Epidemiology Research Office, Department of Internal Medicine, School of

Clinical Medicine, Faculty of Health Sciences, University of the Witwatersrand, Johannesburg

Rebecca Berhanu

Department of Global Health, Boston University School of Public Health

Thandi Dlamini

Right to Care, Johannesburg, South Africa

Lawrence Long

Department of Global Health, Boston University School of Public Health

\section{Denise Evans}

Health Economics and Epidemiology Research Office, Department of Internal Medicine, School of Clinical Medicine, Faculty of Health Sciences, University of the Witwatersrand, Johannesburg

\section{Research article}

Keywords: Multi-drug-resistant tuberculosis, rifampicin resistance, South Africa, Pregnancy, HIV, secondline anti-TB treatment

Posted Date: February 2nd, 2021

DOI: https://doi.org/10.21203/rs.3.rs-155326/v1

License: (c) (i) This work is licensed under a Creative Commons Attribution 4.0 International License. Read Full License 


\section{Abstract}

\section{Background:}

Multi-drug resistant and rifampicin-resistant tuberculosis (MDR/RR-TB) in pregnant women is a cause for concern globally; few data have described the safety of second-line anti-TB medications during pregnancy. We characterize maternal, and pregnancy outcomes for pregnant women receiving secondline anti-tuberculosis treatment for MDR/RR-TB.

\section{Methods:}

We conducted a retrospective record review of pregnant women ( $\geq 18$ years) who received treatment for MDR/RR-TB between 01/2010-08/2016 at three outpatient treatment sites in Johannesburg, South Africa. Demographic, treatment and pregnancy outcome data were collected from available medical records. Preterm birth (<37 weeks), and miscarriage were categorized as adverse pregnancy outcomes.

\section{Results:}

Out of 720 women of child-bearing age who received MDR/RR-TB treatment at the three study sites, 35 (4.4\%) pregnancies were identified. Overall, $68.7 \%$ (24/35) were HIV infected, 83.3\% were on ART. Most women (88.6\%) were pregnant at the time of MDR/RR-TB diagnosis and four women became pregnant during treatment.

Pregnancy outcomes were available for 20/35 (57.1\%) women, which included 15 live births (11 occurred prior to 37 weeks), 1 neonatal death, 1 miscarriage and 3 pregnancy terminations. Overall, 13/17 (76.5\%) had an adverse pregnancy outcome. Of the 28 women with known TB treatment outcomes 17 (60.7\%) completed treatment successfully ( 4 were cured and 13 completed treatment), 3 (10.7\%) died and 8 (28.6\%) were lost-to-follow-up.

\section{Conclusions:}

Pregnant women with MDR/RR-TB suffer from high rates of adverse pregnancy outcomes and about $60 \%$ achieve a successful TB treatment outcome. These vulnerable patients require close monitoring and coordinated obstetric, HIV and TB care.

\section{Background}

Multi-drug resistant and rifampicin-resistant tuberculosis (MDR/RR-TB) remains a significant threat to global control of tuberculosis (TB). An estimated 465000 new cases and 182000 deaths from MDR/RRTB were reported globally in 2018 , with only $56 \%$ of cases successfully treated $(1,2)$. South Africa ranks among the top ten countries in the world in terms of the number of patients with MDR/RR-TB, which is driven by a high HIV burden $(2,3)$. In 2018, South Africa had 13199 MDR/RR-TB cases detected, $72.4 \%$ of 
these started on treatment and treatment success was similar to the global rate, and mortality was above $20 \%(2)$.

An estimated $80 \%$ of women initiating second-line anti-TB treatment for MDR/RR-TB in South Africa are of reproductive age (defined as aged $15-44$ ), and $68.0 \%$ are also HIV-positive (4). TB and HIV co-infection in pregnancy is a major risk factor for maternal mortality and poor neonatal outcomes (4). Multiple drugs used in MDR/RR-TB regimens are known to be potentially teratogenic to the foetus, including aminoglycosides and ethionamide (5). Also, there is limited data from animal studies that linezolid, fluoroquinolones, clofazimine, terizidone and delamanid could be potentially unsafe in pregnancy. However, there is minimal human data as pregnant/breastfeeding women are routinely excluded from clinical trials $(6,7)$. However, the potential risk associated with MDR/RR-TB treatment to the foetus must be weighed against delayed treatment which risks obstetric and neonatal complications, on-going transmission, treatment failure and amplification of resistance from sub-optimal regimens (6-8). Current South African and World Health Organization (WHO) guidelines for the treatment of MDR/RR-TB recommend initiating standard short and long-course regimens for MDR/RR-TB in pregnant and breastfeeding women with the exclusion of aminoglycosides and ethionamide $(9,10)$.

During the study period (2010-2016), the standard of care for MDR/RR-TB treatment in South Africa was a regimen of 5 drugs (kanamycin, moxifloxacin, ethionamide, terizidone, and pyrazinamide) for six months (intensive phase) followed by 18 months (continuation phase) of 4 drugs (oral moxifloxacin, ethionamide, terizidone, and pyrazinamide). For patients with documented resistance or intolerance to the drugs in the standard regimen (e.g. preXDR or XDR-TB), an individualized regimen containing linezolid, para-aminosalicylic acid, clofazimine, and/or capreomycin was used $(11,12)$. In September 2018 , the South Africa National TB program eliminated injectables and recommended the use of all-oral regimens, including for pregnant women $(13,14)$. However, it will be some time before TB treatment, and pregnancy outcomes for women treated with these new regimens are available given the relatively low incidence of pregnancy during MDR/RR-TB treatment. Meanwhile, data on TB treatment and pregnancy outcomes for women on older long course MDR/RR-TB regimens are still extremely valuable for patients and clinicians as both weigh the safety of second-line TB medications during pregnancy.

The aim of the study is to describe TB treatment and pregnancy outcomes among pregnant women receiving second-line anti-tuberculosis treatment for MDR/RR-TB in Johannesburg, South Africa.

\section{Methods}

\section{MDR/RR-TB description}

MDR-TB is TB that is resistant to at least isoniazid (INH) and rifampicin (RIF), the two most important anti-TB drugs in the first-line treatment regimen $(3,15)$. TB that is resistant to RIF but with unidentified or awaiting sensitivities to additional drugs is referred to as rifampicin-resistant TB (RR-TB). MDR-TB with additional resistance to second-line drugs from the fluoroquinolone and injectable drug classes is defined 
as extensively drug-resistant TB (XDR-TB), while preXDR TB is MDR-TB which also has resistance to either a fluoroquinolone or a second-line injectable drug $(15,16)$.

\section{Study setting and design}

We conducted a retrospective record review of adult (18-49 years old) women diagnosed with laboratoryconfirmed MDR/RR-TB, who initiated second-line anti-TB treatment (defined as a regimen containing at least two second-line agents, including at least one of a fluoroquinolone or second-line injectable agent), between 01/2010-08/2016 at three public outpatient treatment sites in Johannesburg, South Africa and had a pregnancy overlap with their TB treatment (Fig. 1). Two of the three sites are decentralized drugresistant TB treatment sites and the third the only specialized hospital for the management of MDR-TB and XDR-TB cases in the Gauteng Province. Eligible participants were identified through each site's respective electronic data management system and MDR/RR-TB registers.

\section{Data Collection}

Clinical data on all eligible women were collected from medical records at treatment sites. This included medical, obstetric, drug-exposure histories, treatments and laboratory data for acute and chronic conditions were collected from medical records at treatment sites. Medical records were defined as all electronic or paper documentation of the patient's medical care at the treatment facilities, including National Health Laboratory Services (NHLS) laboratory reports, prescriptions, MDR/RR-TB patient card, MDR/RR-TB clinic card, antenatal care (ANC) and delivery records and each site's respective electronic data management system, hospital admission records, and maternal or neonatal death records where applicable.

\section{Study variables}

We collected the following patient sociodemographic characteristics at treatment initiation; age (18-29, $\geq 30$ years), nationality (South African or non-South Africa), marital status (in a relationship/married, not in a relationship/widowed), highest education level (< grade 12 versus $\geq$ grade 12) and employment status (employed or unemployed). MDR/RR-TB related information collected include year of MDR/RR-TB treatment initiation (2010-2011, 2012-2013, 2014-2016), MDR/RR-TB treatment regimen and MDR/RRTB treatment regimen changes during the course of treatment. Additionally, we collected TB drugresistance profile (RR-TB, MDR-TB, pre-XDR-TB, XDR-TB), patient category (new, previously treated), and classification of disease (pulmonary, extra-pulmonary, pulmonary and extra-pulmonary).

We categorized pregnancy onset as before or after MDR/RR-TB treatment initiation. Among those pregnant after the MDR/RR-TB treatment initiation, we calculated duration on second-line anti-TB treatment as the time (days) from the start of MDR/RR-TB treatment to the self-reported estimated date of pregnancy onset.

We categorized participants' HIV status and ART status collected from medical records as (HIV-negative, HIV-positive), (on ART, not on ART, ART status unknown) respectively, and collected ART regimens for HIV- 
positive participants initiated on ART. Additionally, ART initiation was categorized as before or after MDR/RR-TB treatment initiation depending on the timing of ART initiation.

\section{Maternal Adverse events}

Adverse events during MDR/RR-TB treatment were determined from laboratory results, patient self-report or clinician documentation of adverse events on patient medical records. Loss of weight, dizziness, rash, nausea and ototoxicity and the severity grade were identified and classified as documented by the clinician. Nephrotoxicity, hepatotoxicity, anaemia, hypokalaemia and neutropenia were confirmed by laboratory tests. Adverse events confirmed by laboratory results were graded using the Division of AIDS (DAIDS) adverse event' categorization (17).

\section{Maternal MDR/RR-TB treatment outcomes}

MDR/RR-TB outcomes were defined using standard TB outcomes as defined in the WHO definitions and reporting framework for TB as cured, completed, died, failed, lost to follow-up (LTFU), or not evaluated (16).

\section{Pregnancy outcomes}

Pregnancy outcomes were assigned in patient medical records according to the standard categories as live birth, miscarriage, stillbirth, and termination of pregnancy $(18,19)$. Preterm birth $(<37$-week gestation), stillbirth, and miscarriage were categorized as adverse pregnancy outcomes. Women were referred to other facilities for antenatal care and delivery but we did not have access to these records. We relied on documentation of antenatal, delivery and neonatal outcomes in the TB patient record. There is no infant outcome classification proposed as infant outcomes were not available in the records.

\section{Analysis}

We used descriptive statistics to summarize demographic, clinical characteristics, pregnancy and TB treatment outcomes. We describe the frequency and severity of adverse events occurring during MDR/RRTB treatment. Continuous variables were described using medians and interquartile ranges (IQR) where appropriate. Categorical variables are described using frequencies and percentages.

Differences by HIV status were determined using the Chi-square or Fisher's exact tests and continuous variables by t-test or Wilcoxon sign-rank-sum test where appropriate. Statistical significance level was set at the $5 \%$ level.

Analysis was conducted using STATA version 14 (Stata Corp, College Station, Texas USA).

The study was approved by the Human Research Ethics Committee (Medical) of the University of the Witwatersrand (Wits HREC M170644). A waiver of informed consent was granted as the study was a retrospective medical record review of routinely collected data.

\section{Results}




\section{Sample selection}

Figure 2 provides a summary of the screening process for sample selection. Of the 715 women aged (15-49 years) initiated on treatment for MDR/RR-TB at the study sites,35 women (5.0\%) were included in the study. We describe the characteristics and outcomes of these 35 women.

\section{Maternal demographic and TB related characteristics}

The median age at MDR/RR-TB treatment initiation was 30 years (IQR:25.0-35.0) (Table 1). A total of $19 / 35$ (54.3\%) of study participants were not in a relationship or were widowed. Overall, 8/35 (22.9\%) of the women completed high school; however, only a quarter (25.7\%) were employed. 
Table 1

Maternal TB and pregnancy characteristics at start of TB treatment by HIV status $(n=35)$

\begin{tabular}{|c|c|c|c|c|}
\hline & Total & $\begin{array}{l}\text { HIV Negative (n } \\
=11 \text { ) }\end{array}$ & $\begin{array}{l}\text { HIV Positive } \\
(n=24)\end{array}$ & $\begin{array}{l}\mathrm{p}- \\
\text { value }\end{array}$ \\
\hline & $\mathrm{N}(\%)$ & $\mathrm{N}(\%)$ & $\mathrm{N}(\%)$ & \\
\hline Age, Med (IQR) & $\begin{array}{l}30(25.0- \\
31.0)\end{array}$ & $27.0(24.0-35.0)$ & $\begin{array}{l}30.1(28.0- \\
35.0)\end{array}$ & \\
\hline $18-29$ & $16(45.7)$ & $7(43.8)$ & $9(56.2)$ & 0.150 \\
\hline$\geq 30$ & $19(54.3)$ & $4(21.1)$ & $15(78.9)$ & \\
\hline \multicolumn{5}{|l|}{ Marital status } \\
\hline In a relationship/married & $7(20.0)$ & $4(36.4)$ & $3(12.5)$ & 0.082 \\
\hline Not in a relationship/widowed & $19(54.3)$ & $3(27.3)$ & $16(66.7)$ & \\
\hline Missing & $9(25.7)$ & $4(36.4)$ & $5(20.8)$ & \\
\hline \multicolumn{5}{|l|}{ Employment } \\
\hline Employed & $9(25.7)$ & $2(18.2)$ & $7(27.2)$ & 0.442 \\
\hline Unemployed & $21(60.0)$ & $6(54.5)$ & $15(62.5)$ & \\
\hline Missing & $5(14.3)$ & $3(27.3)$ & $2(8.3)$ & \\
\hline \multicolumn{5}{|l|}{ Highest education level } \\
\hline Less than Grade 12 & $9(25.7)$ & $3(27.3)$ & $6(25.0)$ & 0.367 \\
\hline Grade 12 and above & $8(22.9)$ & $4(36.4)$ & $4(16.7)$ & \\
\hline Missing & $18(51.4)$ & $4(36.4)$ & $14(58.3)$ & \\
\hline \multicolumn{5}{|l|}{ Nationality } \\
\hline South African & $30(85.7)$ & $8(72.7)$ & $22(91.7)$ & 0.200 \\
\hline Non-South African & $2(5.7)$ & $1(9.1)$ & $1(4.2)$ & \\
\hline Missing & $3(8.6)$ & $2(18.2)$ & $1(4.2)$ & \\
\hline \multicolumn{5}{|l|}{$\begin{array}{l}\text { Year of MDR/RR-TB treatment } \\
\text { registration }\end{array}$} \\
\hline $2010-2011$ & 8 (22.9) & 2 (18.2) & $6(25.0)$ & 0.578 \\
\hline $2012-2013$ & $16(45.7)$ & $4(36.4)$ & $12(50.0)$ & \\
\hline
\end{tabular}

$T B$, tuberculosis; PTB, pulmonary TB; EPTB, extra-pulmonary TB; RR-TB, rifampicin-resistant TB, MDR$T B$, multidrug-resistant TB; XDR-TB, extensively drug-resistant TB; Med, median; IQR, interquartile range 


\begin{tabular}{|c|c|c|c|c|}
\hline & Total & $\begin{array}{l}\text { HIV Negative ( } \\
=11 \text { ) }\end{array}$ & $\begin{array}{l}\text { HIV Positive } \\
(n=24)\end{array}$ & $\begin{array}{l}\mathrm{p}- \\
\text { value }\end{array}$ \\
\hline 2014-2016 & $11(31.4)$ & $5(45.5)$ & $6(25.0)$ & \\
\hline Baseline CD4, cells $/ \mathrm{mm}^{3} \mathrm{Med}(\mathrm{IQR})$ & - & - & $145(51-301)$ & - \\
\hline$<50$ & - & - & $4(16.7)$ & - \\
\hline $51-250$ & - & - & $9(37.5)$ & - \\
\hline$>250$ & - & - & $6(25.0)$ & - \\
\hline Missing & - & - & $5(20.8)$ & - \\
\hline \multicolumn{5}{|l|}{ ART status } \\
\hline On ART & - & - & $20(83.3)$ & - \\
\hline Unknown status & - & - & $4(16.7)$ & - \\
\hline \multicolumn{5}{|l|}{$\begin{array}{l}\text { HIV positive on ART before TB } \\
\text { treatment }\end{array}$} \\
\hline Yes & - & - & $12(60.0)$ & - \\
\hline No & - & - & $6(30.0)$ & - \\
\hline Unknown & & & $2(10.0)$ & - \\
\hline \multicolumn{5}{|l|}{ ART regimen } \\
\hline FDC (TDF + 3TC/FTC + EFV/NVP) & - & - & $8(40.0)$ & - \\
\hline d4T/AZT-3TC-EFV/NVP & - & - & $8(40.0)$ & - \\
\hline d4T-3TC-LPV/r & - & - & $1(5.0)$ & - \\
\hline TDF-3TC-LPV/r & - & - & $1(5.0)$ & - \\
\hline FTC-d4T-EFV & - & - & $1(5.0)$ & - \\
\hline FTC & & & $1(5.0)$ & - \\
\hline \multicolumn{5}{|l|}{ Patient category } \\
\hline New & $19(54.3)$ & $6(54.5)$ & $13(54.2)$ & 0.419 \\
\hline Previously treated & $13(37.1)$ & $3(27.3)$ & $10(41.7)$ & \\
\hline Unknown & $3(8.6)$ & $2(18.2)$ & $1(4.2)$ & \\
\hline
\end{tabular}

$T B$, tuberculosis; PTB, pulmonary TB; EPTB, extra-pulmonary TB; RR-TB, rifampicin-resistant TB, MDR$T B$, multidrug-resistant TB; XDR-TB, extensively drug-resistant TB; Med, median; IQR, interquartile range 


\section{Total}

$\begin{array}{ll}\begin{array}{l}\text { HIV Negative ( } \\ =11)\end{array} & \text { HIV Positive } \\ & (n=24)\end{array}$

p-
value

Previously treated TB resistance profile

$\begin{array}{lllll}\text { RR-TB } & 2(15.4) & - & 2(20.0) & 1.000 \\ \text { MDR-TB } & 6(46.2) & 2(66.7) & 4(40.0) & \\ \text { Missing } & 5(38.5) & 1(33.3) & 4(40.0)\end{array}$

\section{Previously treated TB treatment outcomes}

\begin{tabular}{lllll} 
Completed & $6(46.2)$ & - & $6(60.0)$ & 0.031 \\
Cured & $2(15.4)$ & - & $2(20.0)$ & \\
Treatment failure & $4(30.8)$ & $3(100.0)$ & $1(10.0)$ & \\
Unknown & $1(7.7)$ & - & $1(10.0)$ & \\
TB disease type & & & $13(54.2)$ & 0.452 \\
\hline PTB & $22(62.9)$ & $9(81.8)$ & $7(29.2)$ & \\
EPTB & $9(25.7)$ & $2(18.2)$ & $3(12.5)$ & \\
\hline Both PTB and EPTB & $3(8.6)$ & - & $1(4.1)$ & \\
Missing & $1(2.9)$ & - & $16(66.7)$ \\
Resistance profile & & & $7(29.2)$ \\
\hline RR-TB & $18(51.4)$ & $2(18.2)$ & - \\
\hline MDR-TB & $12(34.3)$ & $5(45.4)$ & $1(4.1)$
\end{tabular}

\section{TB treatment regimen}

$\begin{array}{lllll}\text { Standard second-line TB regimen } & 10(28.6) & 4(36.4) & 6(25.0) & 0.490 \\ \begin{array}{l}\text { Individualized regimen with second- } \\ \text { line drugs }\end{array} & 25(71.4) & 7(63.6) & 18(75.0) & \end{array}$

\section{Pregnant at MDR/RR-TB treatment initiation}

$T B$, tuberculosis; PTB, pulmonary TB; EPTB, extra-pulmonary TB; RR-TB, rifampicin-resistant TB, MDR$T B$, multidrug-resistant TB; XDR-TB, extensively drug-resistant TB; Med, median; IQR, interquartile range 


\begin{tabular}{|lllll|}
\hline & Total & $\begin{array}{l}\text { HIV Negative }(\mathbf{n} \\
\mathbf{1}\end{array}$ & $\begin{array}{l}\text { HIV Positive } \\
(\mathbf{n}=\mathbf{2 4})\end{array}$ & $\begin{array}{l}\text { p- } \\
\text { value }\end{array}$ \\
\hline Yes & $31(88.6)$ & $9(81.8)$ & $22(91.7)$ & 0.575 \\
\hline No & $4(11.4)$ & $2(18.2)$ & $2(8.3)$ & \\
\hline Time to pregnancy onset, Med (IQR) & $\begin{array}{l}10.9(5.5- \\
16.4)\end{array}$ & $5.5(4.0-7.1)$ & $\begin{array}{l}16.4(14.7- \\
18.1)\end{array}$ & 0.121 \\
\hline $\begin{array}{l}\text { TB, tuberculosis; PTB, pulmonary TB; EPTB, extra-pulmonary TB; RR-TB, rifampicin-resistant TB, MDR- } \\
\text { TB, multidrug-resistant TB; XDR-TB, extensively drug-resistant TB; Med, median; IQR, interquartile } \\
\text { range }\end{array}$ & & & & \\
\hline
\end{tabular}

A total of 24 (68.6\%) women were HIV-positive, the median CD4 at ART initiation among women with baseline CD4 counts on file $(n=19)$, was 145 cells/mm3, IQR $(51-301)$. Twenty $(83.3 \%)$ of the HIVpositive women were initiated on ART with $60 \%$ initiated on ART before the start of MDR/RR-TB treatment. While four women had no data regarding ART initiation.

The majority of the women $31 / 35$ (88.6\%) were pregnant at the time of MDR/RR-TB treatment initiation. Four women became pregnant at a median time of 10.9 months IQR (5.5-16.4) after MDR/RR-TB treatment initiation, with HIV-negative women becoming pregnant earlier, at a median time of 5.5 months IQR (4.0-7.1).

More than $50 \%$ of the women were resistant to rifampicin only (RR-TB), $34 \%$ were resistant to rifampicin and other drugs (MDR-TB), and 2 HIV-negative women had XDR-TB. The majority of second-line anti-TB drug regimens were individualized, with only $28.6 \%$ of women receiving standard second-line regimens. Pyrazinamide and terizidone were the most commonly used drugs in the initial treatment regimen in the study cohort (Fig. 3). 
Table 2

Frequency and severity of adverse drugs observed during MDR/RR-TB treatment

\begin{tabular}{|lllll|}
\hline & $\begin{array}{l}\text { Total } \\
(\mathbf{n = 3 5})\end{array}$ & HIV Negative $(\mathbf{n = 1 1 )}$ & $\begin{array}{l}\text { HIV Positive } \\
(\mathbf{n}=\mathbf{2 4})\end{array}$ & p-value \\
\hline Adverse events & & $\mathrm{N}(\%)$ & \\
\hline No adverse events & $18(51.4)$ & $9(81.8)$ & $9(37.5)$ & 0.027 \\
\hline Adverse events & $17(48.6)$ & $2(18.2)$ & $15(62.5)$ & \\
\hline 1 event & $14(82.4)$ & $2(18.2)$ & $12(50.0)$ & \\
\hline$\geq 2$ events & $3(17.6)$ & - & $3(12.5)$ & \\
\hline TB regimen change & & & $9(37.5)$ & 0.656 \\
\hline No & $14(40.0)$ & $5(45.5)$ & $15(62.5)$ & \\
\hline Yes & $21(60.0)$ & $6(54.5)$ & & \\
\hline TB, tuberculosis & & & & \\
\hline
\end{tabular}

Overall, 17/35 (48.6\%) women experienced adverse events during their MDR/RR-TB treatment while also pregnant. The majority, $82.4 \%$, experienced only one adverse event while $17.6 \%$ experienced two or more adverse events. Adverse events were more common in HIV-positive (62.5\%) vs HIV-negative (18.2\%) women. The most prevalent adverse event reported was nephrotoxicity $6 / 17(35.3 \%)$, of which $(2 / 6)$ $33.3 \%$ were moderate (Fig. 4). This was followed by anaemia 4/17 (23.5\%), hypokalaemia 4/17 (23.5\%) and equal number $2 / 17(11.8 \%)$ experiencing ototoxicity or rash. Hypothyroidism, weight loss and dizziness were less common $1 / 17$ (5.9\%) respectively.

\section{Differences in characteristics by HIV status}

HIV-negative patients were different from HIV-positive patients in terms of previous TB treatment outcomes, current TB infection profile and occurrence of adverse events (Table 2). A higher proportion of HIV-negative women had a history of TB treatment failure (100\% vs $11.1 \%, \mathrm{p}<0.05)$ and a higher proportion were infected with MDR-TB and XDR-TB, $(55.6 \%, 22.2 \%$, vs $30.4 \%, 0 \%, p<0.05)$, while more HIV-positive patients presented with RR-TB infection ( $69.6 \%$ vs $22.2 \%, p<0.05)$. Furthermore, HIV-negative women experienced fewer adverse events $(18.2 \%$ vs $62.5 \% ; p<0.05)$.

\section{Maternal TB treatment and pregnancy outcomes}

Seven $(20.0 \%)$ of the women did not have outcomes assigned because they were transferred out of the study site for continued care. Among women with outcomes assigned, 17/28 (60\%) completed treatment, 
of these 4 were cured, whereas $28.6 \%$ women were lost to follow-up, and $10.7 \%$ died (Table 3 ).

Table 3

Maternal TB and pregnancy outcomes by HIV status $(n=35)$

\begin{tabular}{|c|c|c|c|c|}
\hline & Total $(n=28)$ & HIV Negative $(n=8)$ & HIV Positive $(n=20)$ & p-value \\
\hline & $\mathrm{N}(\%)$ & $\mathrm{N}(\%)$ & $\mathrm{N}(\%)$ & \\
\hline \multicolumn{5}{|l|}{ TB treatment outcomes } \\
\hline Completed & $13(46.4)$ & $3(37.5)$ & $10(50.0)$ & 0.759 \\
\hline Cured & $4(14.3)$ & $2(25.0)$ & $2(10.0)$ & \\
\hline Treatment failure & - & - & - & \\
\hline LTFU & $8(28.6)$ & $2(25.0)$ & $6(30.0)$ & \\
\hline \multirow[t]{2}{*}{ Died } & $3(10.7)$ & $1(12.5)$ & $2(10.0)$ & \\
\hline & Total $(n=20)$ & HIV Negative $(n=5)$ & HIV positive $(n=15)$ & p-value \\
\hline Pregnancy outcomes & $\mathrm{N}(\%)$ & $\mathrm{N}(\%)$ & $\mathrm{N}(\%)$ & \\
\hline Live birth (full term) & $4(20.0)$ & $2(40.0)$ & $2(13.3)$ & 0.144 \\
\hline Preterm birth & $11(55.0)$ & $1(20.0)$ & $10(66.7)$ & \\
\hline Miscarriage & $1(5.0)$ & - & $1(6.7)$ & \\
\hline Neonatal death & $1(5.0)$ & - & $1(6.7)$ & \\
\hline Termination & $3(15.0)$ & $2(40.0)$ & $1(6.6)$ & \\
\hline
\end{tabular}

Pregnancy outcomes were available for 20/35 (57.1\%) women. There were 15 live births documented (11 preterm), one miscarriage, one neonatal death and three pregnancy terminations. Overall, 13/20 (65.0\%) women had an adverse pregnancy outcome. TB treatment and pregnancy outcomes did not differ by HIV status among those with known outcomes.

\section{Discussion}

In this cohort of pregnant women receiving second-line anti-TB treatment for MDR/RR-TB in Johannesburg, South Africa, almost two thirds (65\%) of the women experienced adverse pregnancy outcomes including preterm birth (55\%), neonatal death (5\%) and miscarriage (5\%). Adverse pregnancy outcomes in our cohort were more prevalent than the 48\% reported in KwaZulu-Natal province in South Africa among pregnant women managed for MDR/RR-TB from 1 January 2013 to 31 December 2017 (13). The reported preterm births in our cohort were also higher than what was found in the KwaZulu- 
Natal cohort and a cohort of 38 pregnant women treated for MDR/RR-TB in Lima, Peru between 1996 and $2005(13,20)$. Most concerning is that the preterm births in our cohort was also higher than in HIV and general populations in South Africa $(13,20-24)$. Almost all of the adverse birth outcomes were among HIV-positive women in our cohort. Highlighting the need for consistent implementation of regular TB screening and Isoniazid preventative therapy (IPT) among HIV-positive pregnant women, which has been proven to be safe and effective (25).

Overall, $60.1 \%$ of the women in our study completed MDR/RR-TB treatment, with $11.4 \%$ cured. These outcomes are comparable to the national drug-resistant TB success of $54 \%$ for South Africa in 2016, and similar to the treatment success rate in the Lima, Peru cohort $(26,27)$. The Lima cohort only had much lower HIV co-infection (7.9\%). The KwaZulu-Natal cohort had slightly better TB outcomes of $67 \%$ treatment success rate. However, half of this cohort received bedaquiline as part of their TB treatment regimen, which has shown improved treatment success rates among MDR/ RR-TB patients (13).

The majority of the women in our study were pregnant at MDR/RR-TB diagnosis. Those that became pregnant after TB treatment initiation became pregnant during the continuation phase of treatment when most patients start to feel better and start resuming normal life activities. The lack of family planning for these patients is a cause for concern as it is specified as part of the management of MDR/RR-TB in women of childbearing age.

Our study is the first to describe the occurrence of adverse events among women treated with second-line TB drugs while pregnant. Almost $48.5 \%$ of the women experienced at least one adverse event, which is lower than $83 \%$ that was reported previously in a systematic review to estimate the prevalence of adverse events during DR-TB treatment among non-pregnant patients (28). Higher rates of adverse events occurred among HIV-positive mothers, likely due to the concurrent use of ART (28). Hepatotoxicity and nephrotoxicity were most prevalent ; they have been linked to pyrazinamide, ethionamide, kanamycin and fluoroquinolones $(29,30)$ which were the most commonly used drugs to treat our study cohort. Aminoglycosides and ethionamide have since been removed from the new all-oral short and long MDR/RR regimens in South Africa, but given the increasing availability of new drugs such as bedaquiline and linezolid their use among pregnant women should be reconsidered for countries still using the long regimen, (1).

\section{Limitations}

The retrospective design might have introduced selection or information bias. Women were referred to other facilities for ANC and delivery; therefore, birth and pregnancy outcomes were poorly documented in the TB records reviewed. Reporting of adverse events was incomplete, and some laboratory result forms were missing from patient files, likely resulting in an underestimation of adverse events. Additionally, since our data relies on the accurate reporting of pregnancy in the medical record, $n=35$ may be an underestimate if not all the pregnancies were recorded. 
Although the data was collected from 3 main hospitals, the sample size was very small; therefore, findings may not be generalizable and applicable to other populations.

\section{Conclusions}

Pregnant women with MDR-TB/RR-TB suffer from high rates of adverse pregnancy outcomes. Results from our study highlight the need for close monitoring and coordinated obstetric, HIV and TB care for these vulnerable patients.

\section{List Of Abbreviations}

ANC Antenatal care

ART Antiretroviral therapy

CD4 Cluster of differentiation 4

DAIDS Division of acquired immunodeficiency syndrome

HIV Human immunodeficiency virus

HREC Human Research Ethics Committee

INH isoniazid

IPT Isoniazid preventative therapy

IQR Interquartile range

LTFU lost to follow-up

MDR/RR-TB Multi-drug resistant and rifampicin-resistant tuberculosis

NHLS National Health Laboratory Services

RIF rifampicin

TB Tuberculosis

WHO World Health Organisation

XDR-TB Extensively drug-resistant tuberculosis

\section{Declarations}




\section{Ethics approval and consent to participate}

The study was approved by the Human Research Ethics committee of the Witwatersrand University, Johannesburg (M170644). The requirement for written informed consent was waived as this was a retrospective analysis of routine medical record data.

\section{Consent for publication}

Not applicable

\section{Availability of data and materials}

The datasets generated and/or analyzed during the current study are not publicly available as the data are owned by the study sites and the National Department of Health (South Africa) and governed by the Human Research Ethics Committee (University of the Witwatersrand, Johannesburg, South Africa). All relevant data are included in the paper. The full data are available from the Health Economics and Epidemiology Research Office for researchers who meet the criteria for access to confidential data and with permission from the owners of the data. Contact the organization at information@heroza.org for additional information regarding data access.

\section{Competing interests}

The authors have declared that no competing interests exist

\section{Funding}

This study has been made possible by the generous support of the American People and the President's Emergency Plan for AIDS Relief (PEPFAR) through USAID under the terms of Cooperative Agreements AID-674-A-12-00029 and 72067419CA00004 to Health Economics and Epidemiology Research Office and under the terms of Cooperative Agreement 674-A-00-09-00018-00 to Boston University. The contents are the responsibility of the authors and do not necessarily reflect the views of PEPFAR, USAID or the United States Government. The funders had no role in the study design, collection, analysis and interpretation of the data, in manuscript preparation or the decision to publish.

\section{Author's contribution}

IM conceptualized and designed the study. IM and NJ conducted the formal analysis and completed the original drafted the manuscript. DE, RB, TD and LL provided feedback on the manuscript. All authors 
assisted in interpreting the results, critically reviewed and approved the final version of the manuscript.

\section{Acknowledgements}

The authors wish to thank the staff at all facilities involved in supporting data collection and verification. We would also like to thank Melda Musina and Busi Sithole for their contributions to this study.

\section{References}

1. World Health Organisation W. Global Tuberculosis Report 2020. Geneva: World Health Organisation 2020.

2. World Health Organization (WHO). Global tuberculosis report 2019. Geneva: World Health Organization, 2019.

3. Cox H, Dickson-Hall L, Jassat W, Moshabela M, Kielmann K, Grant A, et al. Drug-resistant tuberculosis in South Africa: history, progress and opportunities for achieving universal access to diagnosis and effective treatment. South African Health Review. 2017;2017(1):157-67.

4. Schnippel K, Ndjeka N, Conradie F, Berhanu R, Claasen Z, Banoo S, et al. A call to action: Addressing the reproductive health needs of women with drug-resistant tuberculosis. South African Medical Journal. 2016;106(4):333-4.

5. Laniado-Laborín R, Carrera-López K, Hernández-Pérez A. Unexpected Pregnancy during Treatment of Multidrug-resistant Tuberculosis. Turk Thorac J. 2018;19(4):226-7.

6. Mathad JS, Gupta A. Tuberculosis in pregnant and postpartum women: epidemiology, management, and research gaps. Clinical infectious diseases. 2012;55(11):1532-49.

7. Briggs GG, Freeman RK, Yaffe SJ. Drugs in pregnancy and lactation: a reference guide to fetal and neonatal risk: Lippincott Williams \& Wilkins; 2012.

8. Figueroa-Damian R, Arredondo-Garcia J. Pregnancy and Tuberculosis: Influence of Treatment on Perinatal Outcome. American Journal of Perinatology. 1998;15(05):303-6.

9. South African National Department of Health N. Management of Rifampicin-Resisitant Tuberculosis: A Clinical Reference Guide 2019.

10. (WHO); WHO. World Health Organisation Consolidated guidelines on drug-resistant tuberculosis treatment. 2019.

11. National Department of Health Republic of South Africa. Multi-drug resistant tuberculosis: Introduction of New Drugs and Drug Regimens for the Management of Drug-Resistant Tuberculosis in South Africa: Policy Framework. Pretoria 2015.

12. National Department of Health Republic of South Africa. Management of Drug-Resistant Tuberculosis: Policy Guidelines. Pretoria 2011.

13. Loveday M, Hughes J, Sunkari B, Master I, Hlangu S, Reddy T, et al. Maternal and infant outcomes among pregnant women treated for multidrug/rifampicin-resistant tuberculosis in South Africa. 
Clinical Infectious Diseases. 2020.

14. (NDoH); NDoH. INTERIM CLINICAL GUIDANCE FOR THE IMPLEMENTATION OF INJECTABLE-FREE REGIMENS FOR RIFAMPICIN-RESISTANT TUBERCULOSIS IN ADULTS, ADOLESCENTS AND CHILDREN. 2018.

15. World Health Organization (WHO). Companion handbook to the WHO guidelines for the programmatic management of drug-resistant tuberculosis: World Health Organization; 2014.

16. World Health Organization (WHO) Definitions and reporting framework for tuberculosis-2013 revision. 2013.

17. World Health Organization (WHO). The use of the WHO-UMC system for standardized case causality assessment. Uppsala: The Uppsala Monitoring Centre. 2005.

18. National Department of Health RoSA. Guidelines for Maternity Care in South Africa 2007: A manual for clinics, community health centres and district hospitals 2015. 2015.

19. World Health Organization (WHO). International classification of diseases and related health problems: manual of the International Statistical Classification of Diseases, Injuries, and Causes of Death. World Health Organization, Geneva. 1992.

20. Palacios E, Dallman R, Muñoz M, Hurtado R, Chalco K, Guerra D, et al. Drug-resistant tuberculosis and pregnancy: treatment outcomes of 38 cases in Lima, Peru. Clinical infectious diseases. 2009;48(10):1413-9.

21. van de Water BJ, Brooks MB, Huang C-C, Trevisi L, Lecca L, Contreras C, et al. Tuberculosis clinical presentation and treatment outcomes in pregnancy: a prospective cohort study. BMC Infectious Diseases. 2020;20(1):1-8.

22. Simbayi L, Zuma K, Zungu N, Moyo S, Marinda E, Jooste S, et al. South African National HIV Prevalence, Incidence, Behaviour and Communication Survey, 2017: towards achieving the UNAIDS 90-90-90 targets. 2019.

23. Chawanpaiboon S, Vogel JP, Moller A-B, Lumbiganon P, Petzold M, Hogan D, et al. Global, regional, and national estimates of levels of preterm birth in 2014: a systematic review and modelling analysis. The Lancet Global Health. 2019;7(1):e37-e46.

24. Dadabhai S, Gadama L, Chamanga R, Kawalazira R, Katumbi C, Makanani B, et al. Pregnancy outcomes in the era of universal antiretroviral treatment in sub-Saharan Africa (POISE Study). Journal of acquired immune deficiency syndromes (1999). 2019;80(1):7.

25. Gupta A, Montepiedra G, Aaron L, Theron G, McCarthy K, Bradford S, et al. Isoniazid Preventive Therapy in HIV-Infected Pregnant and Postpartum Women. New England Journal of Medicine. 2019;381(14):1333-46.

26. Palacios E, Dallman R, Muñoz M, Hurtado R, Chalco K, Guerra D, et al. Drug-Resistant Tuberculosis and Pregnancy: Treatment Outcomes of 38 Cases in Lima, Peru. Clinical Infectious Diseases. 2009;48(10):1413-9.

27. Organization WH. Global TB Report 2017 (2017). 
28. Schnippel K, Firnhaber C, Berhanu R, Page-Shipp L, Sinanovic E. Adverse drug reactions during drugresistant TB treatment in high HIV prevalence settings: a systematic review and meta-analysis. The Journal of antimicrobial chemotherapy. 2017.

29. Mathews A, Bailie G. Clinical pharmacokinetics, toxicity and cost effectiveness analysis of aminoglycosides and aminoglycoside dosing services. Journal of clinical pharmacy and therapeutics. 1987;12(5):273-91.

30. Buziashvili M, Mirtskhulava V, Kipiani M, Blumberg H, Baliashvili D, Magee M, et al. Rates and risk factors for nephrotoxicity and ototoxicity among tuberculosis patients in Tbilisi, Georgia. The International Journal of Tuberculosis and Lung Disease. 2019;23(9):1005-11.

\section{Figures}

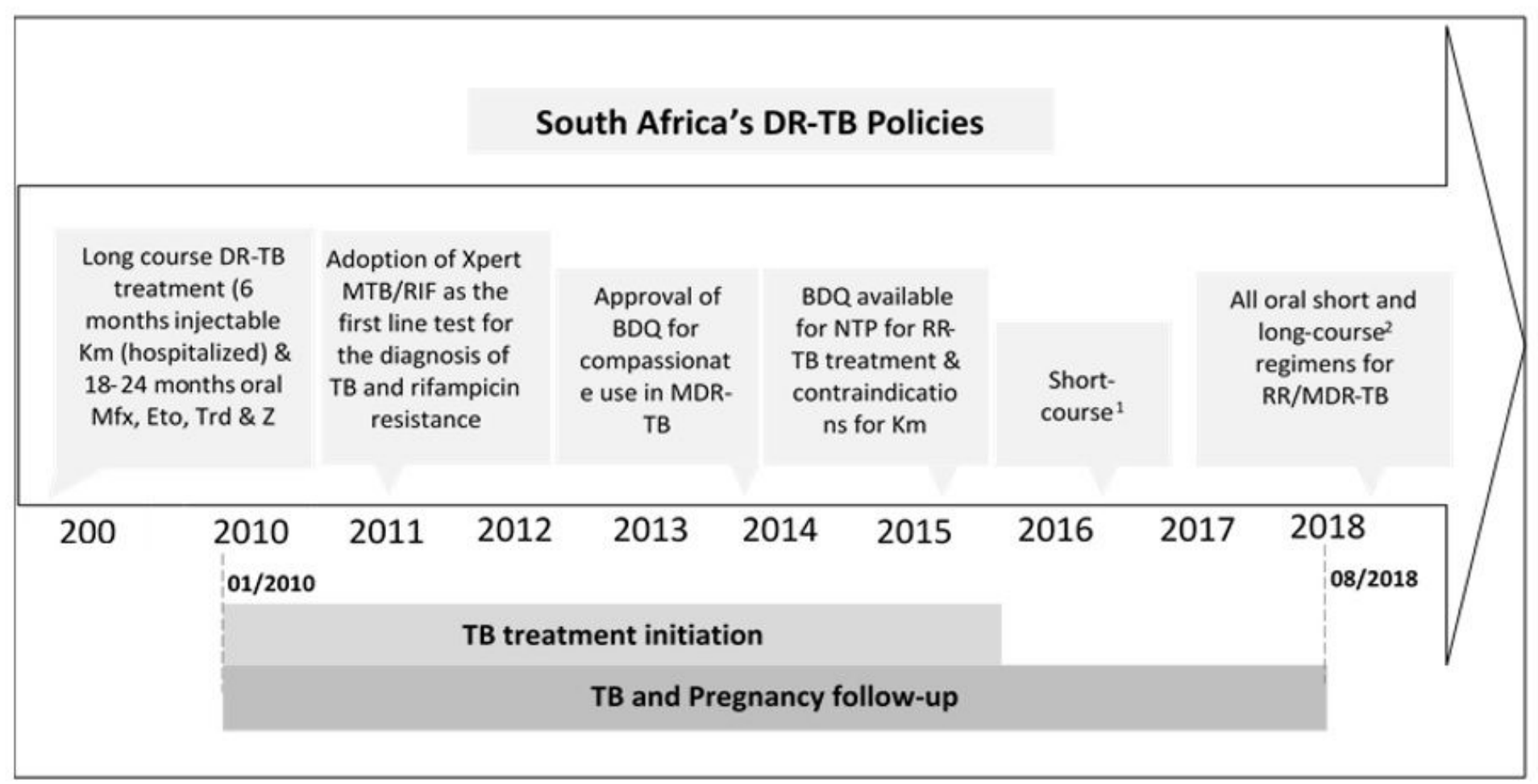

\section{Figure 1}

Study period alongside South Africa's drug-resistant TB policies. Km - Kanamycin, Mfx - Moxifloxacin, Eto - Ethambutol, INH - Isoniazid, Cfz - Clofazimine, CfX - Cefozitin, BDQ - Bedaquiline, Trd Terizidone, Z - Pyrazinamide, NTP- National TB programme 1 Short course: BDQ, LZD, LFX, CFZ, Hi-INH, PZA, EMB x 9-11 months 2 Long course: BDQ, LFX, LZD, TRD and CFZ for 18 months 


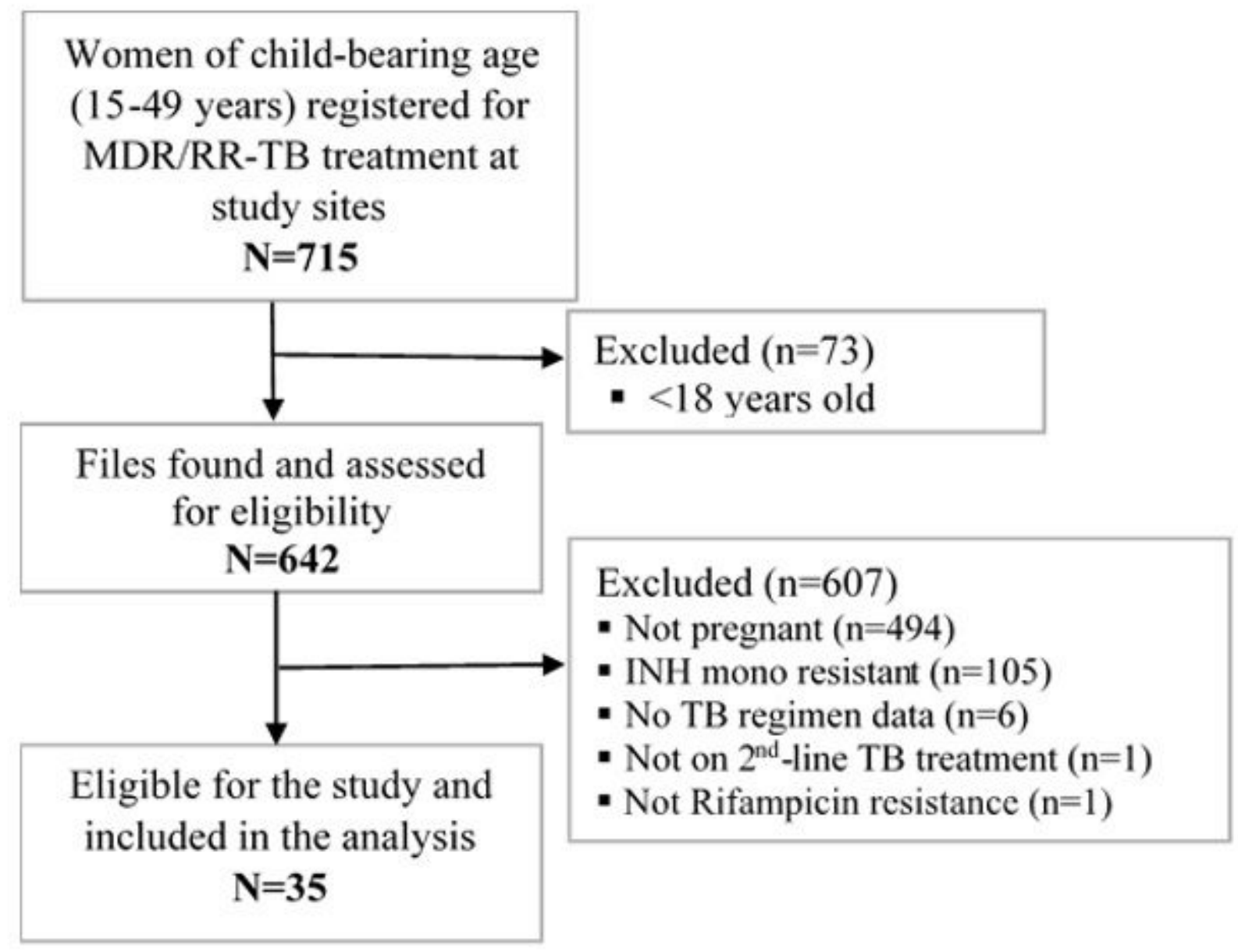

Figure 2

Summary of participant selection 
12

10

8

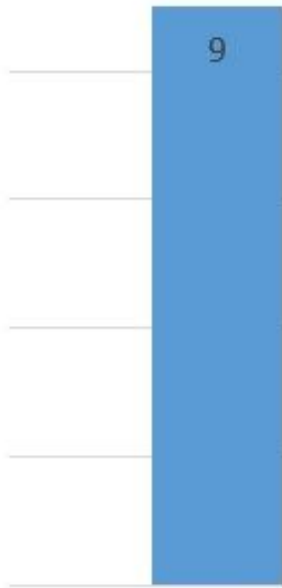

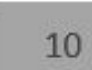
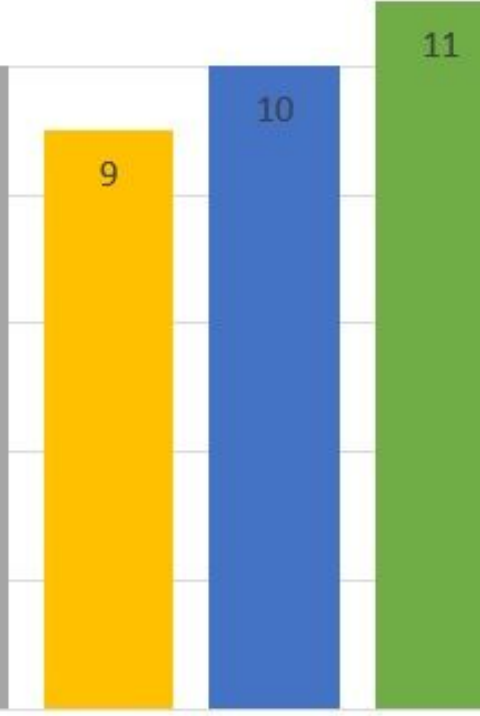

\section{1}

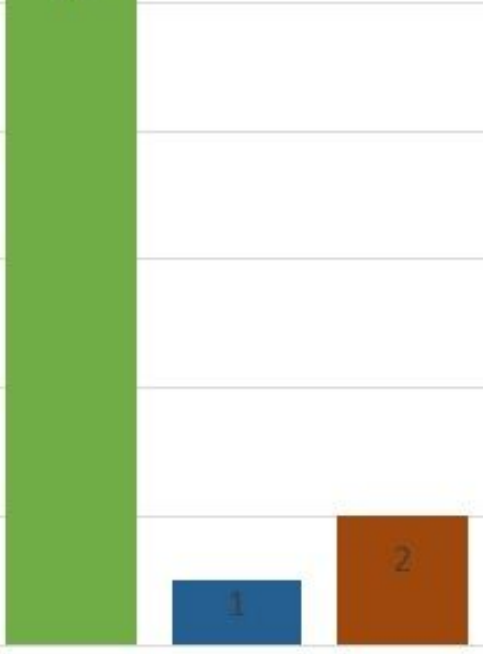

$\square \mathrm{KM} \backsim \mathrm{CM} \| \mathrm{FQ}=\mathrm{ETO}$

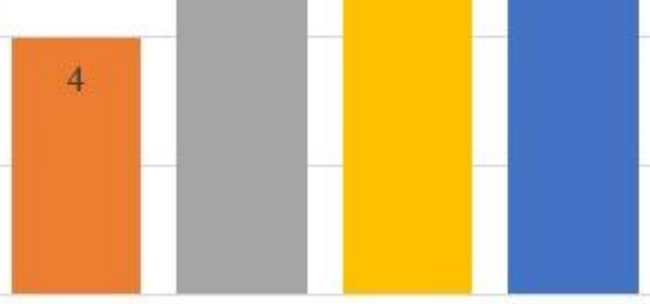

- TRD —PZA =LZD घPAS

\section{Figure 3}

Frequency of anti-TB drugs contained in initial treatment regimens among women pregnant at MDR/RRTB treatment initiation $(n=31) \mathrm{KM}$ - Kanamycin, CM - Capreomycin, FQ - Fluoroquinolones, ETO Ethambutol, TRD - Terizidone, PZA - Pyrazinamide, LZD - Linezolid, PAS - P-aminosalicyclic acid 


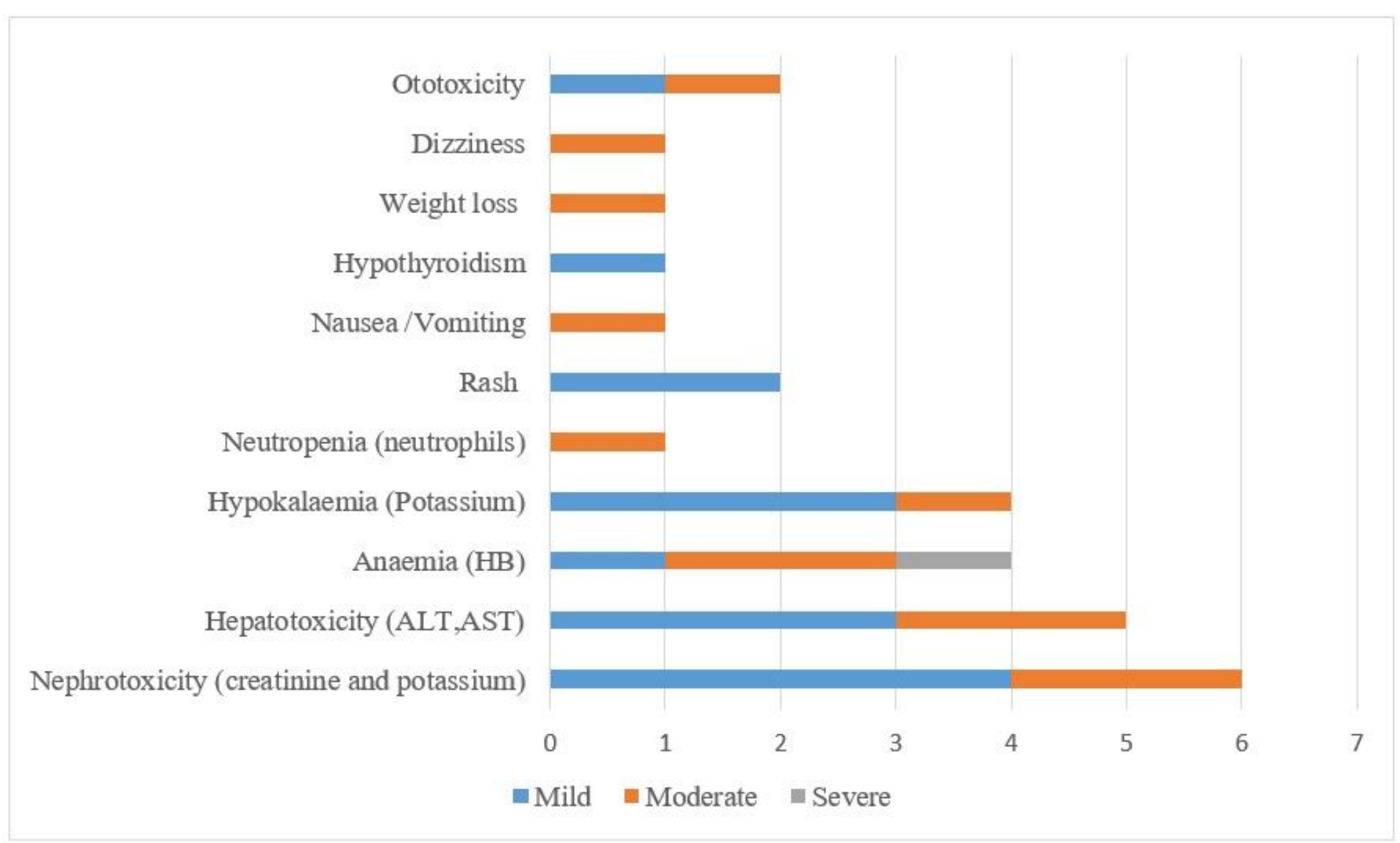

\section{Figure 4}

Frequency and severity of adverse drug observed during RR-TB treatment $(n=17)$. One HIV negative women reported nausea/vomiting and one HIV negative woman reported mild hypokalaemia 